OPEN ACCESS

Edited by:

Pengfei Zhang,

Shenzhen Institutes of Advanced

Technology, Chinese Academy

of Sciences (CAS), China

Reviewed by:

Abbas Amini,

Australian College of Kuwait, Kuwait

Chong Wang,

Dongguan University of Technology,

China

*Correspondence:

Huiqiang Sun

whitedove69@163.com

Specialty section:

This article was submitted to

Nanobiotechnology,

a section of the journa

Frontiers in Bioengineering and

Biotechnology

Received: 18 March 2021

Accepted: 15 June 2021

Published: 15 July 2021

Citation:

Zhang $T$, Jiang $M$, Yin $X$, Yao $P$

and Sun $H$ (2021) Mechanism

of Exosomes Involved

in Osteoimmunity Promoting

Osseointegration Around Titanium

Implants With Small-Scale

Topography.

Front. Bioeng. Biotechnol. 9:682384.

doi: 10.3389/fbioe.2021.682384

\section{Mechanism of Exosomes Involved in Osteoimmunity Promoting Osseointegration Around Titanium Implants With Small-Scale Topography}

\author{
Ting Zhang ${ }^{1}$, Mengyang Jiang ${ }^{1}$, Xiaojie Yin ${ }^{1}$, Peng Yao ${ }^{2}$ and Huiqiang Sun ${ }^{1 *}$ \\ 'Department of Prosthodontics, School and Hospital of Stomatology, Cheeloo College of Medicine, Shandong University \& \\ Shandong Key Laboratory of Oral Tissue Regeneration \& Shandong Engineering Laboratory for Dental Materials and Oral \\ Tissue Regeneration, Jinan, China, ${ }^{2}$ School of Mechanical Engineering, Shandong University, Jinan, China
}

Exosomes are nanoscale extracellular vesicles. Several studies have shown that exosomes participate in intercellular communication and play a key role in osseointegration. However, it is unclear whether exosomes and their contents participate in the communication between the immune and skeletal systems in the process of osseointegration. In this study, we obtained smooth titanium disks by polishing and small-scale topography titanium disks by sandblasted large-grit acidetched (SLA) technology combined with alkali thermal reaction. After stimulating mouse RAW264.7 cells with these two kinds of titanium disks, we co-cultured the MC3T3E1 cells and the RAW264.7 cells, obtained and identified the exosomes derived from RAW264.7 cells, and studied the effect of the osteoimmune microenvironment and the exosomes on the osseointegration of mouse MC3T3-E1 cells. Cell counting kit-8 (CCK8), real time quantitative PCR, western blotting, alizarin red staining, and quantitative and confocal fluorescence microscopy were used to study the effects of exosomes on MC3T3-E1 cells; RNA sequencing and correlation analysis were performed. We found that the osteoimmune microenvironment could promote the osseointegration of MC3T3E1 cells. We successfully isolated exosomes and found that RAW264.7 cell-derived exosomes can promote osteogenic differentiation and mineralization of MC3T3-E1 cells. Through RNA sequencing and gene analysis, we found differentially expressed microRNAs that targeted the signal pathways that may be related, such as mTOR, AMPK, Wnt, etc., and thus provide a reference for the mechanism of osteoimmunue regulation of implant osseointegration. The study further elucidated the mechanism of implant osseointegration and provided new insights into the effect of exosomes on implant osseointegration, and provided reference for clinical improvement of implant osseointegration and implant success rate.

\footnotetext{
Keywords: exosomes, microRNA, osteoimmunity, titanium, osseointegration
} 


\section{INTRODUCTION}

In recent years, implant dentures have gradually become an important treatment option for missing teeth. Titanium and titanium alloys have good biocompatibility and mechanical properties and are among the most widely used implant materials in clinics (Xue et al., 2020). However, because titanium is an inert metal with no biological activity, it can easily cause a host inflammatory reaction that can even progress to chronic inflammation, delaying implant osseointegration (Dar et al., 2018). Therefore, many techniques have been applied for titanium surface modification (Dohan Ehrenfest et al., 2010), such as sandblasted large-grit acid etching (SLA) and anodization (Loi et al., 2016; Miron and Bosshardt, 2016). In our previous study, we combined SLA technology with an alkali thermal reaction to construct titanium implants with small-scale topography. It was found that, small-scale topography promoted MC3T3-E1 cell proliferation and osteogenic differentiation better than the polished smooth surface, the micro-surface obtained by SLA technology and the nano-surface obtained by alkali thermal reaction (Yang et al., 2020).

The process of osseointegration of implants involves the coordinated operation of the immune and skeletal systems, namely osteoimmunity (Dar et al., 2018). Macrophages can be differentiated into resident cells or myeloid precursor cells (mainly monocytes) and reside in the bone. The interaction between macrophages and osteocytes is crucial for bone formation and repair (Pieters et al., 2019). Some studies have found that different implant surface morphologies can induce macrophages to polarize to the pro-inflammatory M1 phenotype or anti-inflammatory M2 phenotype (Luu et al., 2015; Quan et al., 2020). In our previous study, we found that the smallscale topography can stimulate RAW264.7 cells to polarize to anti-inflammatory M2 phenotype and regulate the osteoimmune microenvironment to an anti-inflammatory environment, which is more conducive to implant osseointegration (Yang et al., 2020).

Exosomes are nano-sized vesicles that are secreted by most cells. They were first found in reticulocytes in 1983 and named exosome in 1987 ( $\mathrm{Pan}$ and Johnstone, 1983). The diameter of exosomes is in the range of $30-150 \mathrm{~nm}$, with a lipid bilayer structure (Mathieu et al., 2019); exosomes can be directly absorbed by target cells and affect the phenotype of receptor cells (Rani et al., 2015; Yang et al., 2017). Therefore, they play an important role in cell communication and have attracted increasing attention. Wei et al. (2019) found that the expression of alkaline phosphatase (ALP) and BMP-2 markers of early osteoblast differentiation was significantly increased by using BMP-2/macrophage- derived exosomes to modify titanium nanotube implants, which confirmed that the combination of titanium nanotubes and BMP-2/macrophagederived exosomes could promote bone formation. Xiong et al. (2020) found that M2 macrophage-derived exosome microRNA5106 could induce the osteogenic differentiation of bone marrow mesenchymal stem cells.

Exosomes play an important role in target cells, mainly through intercellular communication and the delivery of key bioactive factors. However, it is not clear whether exosomes participate in osteoimmunity and influence osteointegration around titanium implants with small-scale topography. Furthermore, the gene information and function of macrophagederived exosomes have not been fully clarified. Therefore, we studied the effect of macrophage-derived exosomes stimulated by small-scale topography of titanium implants on MC3T3-E1 cells and screened key microRNAs in exosomes, to further explore the mechanism of macrophages stimulated by exosomal contents in small-scale topography titanium disks on MC3T3-E1 cells, and to provide reference for exploring the effect of osteoimmunity on osseointegration.

\section{MATERIALS AND METHODS}

\section{Preparation and Characterization of Titanium Disk}

Ti6Al4V disks with a diameter of $19.5 \mathrm{~mm}$ and thickness of $1 \mathrm{~mm}$ (Taizhou Yutai Metal Materials Co., Ltd., Jiangsu, China) were used and polished to an average roughness of $0.2 \mathrm{~mm}$ and a thickness of $0.01 \mathrm{~mm}$. The polishing process of the disks was listed in Supplementary File 1. The 60 mesh alumina particles (Gongyi Baolai Water Treatment Material Factory, Henan, China) were sprayed on the polished titanium plate surface at a spray angle of $90^{\circ}$ with a spray distance of no more than $5 \mathrm{~cm}$. When the surface of the titanium disk was uniformly gray, it was removed and immersed in $0.5 \%$ hydrofluoric acid solution for $15 \mathrm{~min}$ at $25^{\circ} \mathrm{C}$. For alkali thermal treatment, the titanium disk was immersed in a $10 \mathrm{~mol} / \mathrm{L}$ sodium hydroxide solution and treated at $80^{\circ} \mathrm{C}$ for $24 \mathrm{~h}$. All the titanium disks were placed into a $5 \%$ concentrated cleaning solution (micro90, International Products Company, New York, United States), anhydrous ethanol, distilled water, ultrasonic vibration cleaning for $5 \mathrm{~min}$, and air dried at room temperature for standby.

For the surface characterization of the titanium disk, the surface morphology was observed using a cold field emission scanning electron microscope (Carl Zeiss, Germany) and 3D laser scanning microscope (VK-X200 K, Japan). The surface contact angle was measured by the suspension drop method with $2 \mu \mathrm{L}$ artificial saliva, and the average surface roughness (RA) of the titanium disk was measured using a Wyko nt9300 optical profiler (Veeco, United States). The surface composition of titanium disks was analyzed by X-ray photoelectron spectrometer (Thermo Fisher Scientific, United States).

\section{Cell Culture}

RAW264.7 cells, a widely used mouse derived macrophage cell line, were provided by Shandong Key Laboratory of oral tissue. Mouse embryonic osteoblast precursor cells (MC3T3-E1 cells) were purchased from the Cell Bank of the Chinese Academy of Sciences (Shanghai, China). The complete medium was $\alpha$-minimum essential medium ( $\alpha$-MEM, Hyclone, United States), in which $10 \%$ fetal bovine serum (FBS, Hyclone, United States) was added. The culture medium contained double antibodies (100 IU/mL penicillin G and $100 \mu \mathrm{g} / \mathrm{mL}$ streptomycin, Solebo, China). The cells were cultured at $37^{\circ} \mathrm{C}$ in a $5 \% \mathrm{CO}_{2}$ incubator, and the medium was changed on alternate days. 
Osteogenesis induction medium (OM) consisted of complete medium supplemented with $50 \mathrm{mg} / \mathrm{L}$ ascorbic acid (Sigma Aldrich), $10 \mathrm{mmol} / \mathrm{L} \beta$-glycerophosphate (Sigma Aldrich), and $10 \mathrm{nmol} / \mathrm{L}$ dexamethasone (Sigma Aldrich). The supernatant of RAW264.7 cell culture medium was centrifuged at $1000 \times g$ for $5 \mathrm{~min}$, and then the conditioned medium (CM) was prepared by adding 20\% FBS osteogenic induction medium.

\section{Exosome Isolation}

The supernatant of RAW264.7 cell culture medium was centrifuged at $1000 \times g$ for $5 \mathrm{~min}$, and then $20 \mathrm{~mL}$ of each group was added into a $50 \mathrm{~mL}$ centrifuge tube. PBS was added to bring it to a total of $40 \mathrm{~mL}$ in each tube, centrifuged at $4^{\circ} \mathrm{C}$ for $10 \mathrm{~min}$ at $300 \times \mathrm{g}$, and the supernatant was transferred to a new $50 \mathrm{~mL}$, the supernatant was transferred to a centrifuge tube $(40 \mathrm{~mL})$, matched with the ultracentrifuge, and centrifuged at $4^{\circ} \mathrm{C}$ for $30 \mathrm{~min}$ at $10,000 \times \mathrm{g}$. The supernatant was transferred to a centrifuge tube $(40 \mathrm{~mL})$, matched with the ultracentrifuge, and centrifuged at $4^{\circ} \mathrm{C}$ for $70 \mathrm{~min}$ at $10,0000 \times g$. The exosomes were identified by transmission electron microscopy, nanoparticle tracking analysis, and western blotting.

\section{Nanoparticle Tracking Analysis (NTA)}

The sample pool was cleaned with deionized water, the instrument was calibrated with polystyrene microspheres $(110 \mathrm{~nm})$, and the sample pool was cleaned with $1 \times$ PBS buffer; the exosome solution was diluted with $1 \times$ PBS buffer and injected for detection. Each sample was detected three times, and the data were processed and mapped using the Origin software.

\section{CCK-8 Detection}

Cell proliferation was examined using the cell counting kit 8 (CCK-8; Dojindo, Tokyo, Japan). MC3T3-E1 cells were treated with osteogenic induction medium or CM. On days 1, 3, 5, and 7 , the original medium was replaced with CCK- 8 reagent and complete medium at a ratio of 1:10. MC3T3-E1 cells were incubated at $37^{\circ} \mathrm{C}$ for $1 \mathrm{~h}$, and the absorbance value was measured at $450 \mathrm{~nm}$.

\section{Alizarin Red S Staining and Cetylpyridinium Chloride Determination}

MC3T3-E1 cells were fixed with $4 \%$ paraformaldehyde for $30 \mathrm{~min}$ and then incubated with alizarin red S (Sigma Aldrich) for $10 \mathrm{~min}$. After washing with deionized water, the calcium deposition was observed using an optical microscope. Cetylpyridinium chloride (10\%; Sigma Aldrich) was used for quantification, and the absorbance value was determined at $562 \mathrm{~nm}$.

\section{Real Time Fluorescent Quantitative PCR (qRT-PCR)}

Total RNA was extracted using TRIzol reagent (Invitrogen, NY, United States). cDNA was synthesized using the PrimeScript RT Master Mix Kit (Takara Biotechnology, China). GAPDH was selected as the internal reference gene, and the relative gene expression was calculated by the " $2-\triangle \triangle \mathrm{CT}$ " method. The primer sequences are listed in Table 1.

\section{Western Blot}

Cells were lysed with Ripa buffer (Wanleibio, China) containing $10 \mathrm{mM}$ protease inhibitor (PMSF; Wanleibio, China). The lysate was centrifuged at $12,000 \mathrm{rpm}$ and $4^{\circ} \mathrm{C}$ for $10 \mathrm{~min}$. The supernatant was separated, and the protein concentration was determined using a BCA protein concentration assay kit (Wanleibio, China). Equivalent amounts of protein (40 g) were added to the $8 \%-15 \%$ SDS-PAGE gel and then transferred to a PVDF membrane (Millipore, Billerica, United States). The membrane was sealed in 5\% skimmed milk powder solution for $1 \mathrm{~h}$, and then incubated with the following primary antibodies: runt-related transcription factor 2 (Runx2, wl03358; Wanleibio, China), Collagen I (wl0088; Wanleibio, China), $\beta$-actin (w101845; Wanleibio, China), CD9 (ab92726, Abcam, United Kingdom), CD63 (ab216130, Abcam, United Kingdom), and TSG101 (ab125011, Abcam, United Kingdom) at $4^{\circ} \mathrm{C}$ overnight. After washing with TBST four times, the membrane was incubated with sheep anti-rabbit IgG HRP (wla023; Wanleibio, China) at room temperature for $45 \mathrm{~min}$. An ECL detection kit (Wanleibio, China) was used to visualize the protein bands. Proteins on the same membrane were compared quantitatively by determining the optical density of the target strip using a gel image processing system (Gel-Pro-Analyzer software, Media Cybernetics, United States).

\section{Exosome RNA Sequencing and Gene Analysis}

According to the standard procedure provided by Illumina (San Diego, United States), the miRNA sequencing library was prepared using the TruSeq Small RNA Sample Prep Kit (Illumina, San Diego, United States). After library preparation, the constructed library was sequenced using Illumina HiSeq2000/2500, and the reading length was $1 \times 50 \mathrm{bp}$. Clean reads were obtained from original data after quality control. The $3^{\prime}$-linker was removed from the clean reads, and the length of the $3^{\prime}$ linker was screened. Sequences with a base length of 18-25 nt (plant) or 18-26 nt (animal) were retained. The remaining sequences were then aligned to various RNA

TABLE 1 | List of primers used in this study for qRT-PCR.

\begin{tabular}{|c|c|c|}
\hline Genes & Primers & Sequences $\left(5^{\prime}-3^{\prime}\right)$ \\
\hline \multirow[t]{2}{*}{ GAPDH } & Forward & AGGTCGGTGTGAACGGATाG \\
\hline & Reverse & TGTAGACCATGTAGTTGAGGTCA \\
\hline \multirow[t]{2}{*}{ IL-1 $\beta$} & Forward & GTGTCTTCCCGTGGACCTा \\
\hline & Reverse & AATGGGAACGTCACACACCA \\
\hline \multirow[t]{2}{*}{$\mathrm{IL}-10$} & Forward & GCTCTTGCACTACCAAAGCC \\
\hline & Reverse & CTGCTGATCCTCATGCCAGT \\
\hline \multirow[t]{2}{*}{ Runx2 } & Forward & GGGACTGTGGTTACCGTCAT \\
\hline & Reverse & ATAACAGCGGAGGCATाTCG \\
\hline \multirow[t]{2}{*}{ Collagen I } & Forward & CCСTGGTCCСTCTGGAAATG \\
\hline & Reverse & GGACCTाGCCCCCПTCПT \\
\hline
\end{tabular}



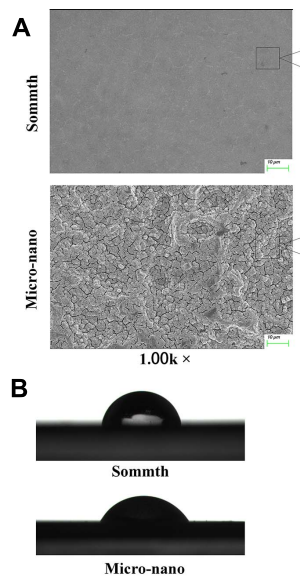

Micro-nano
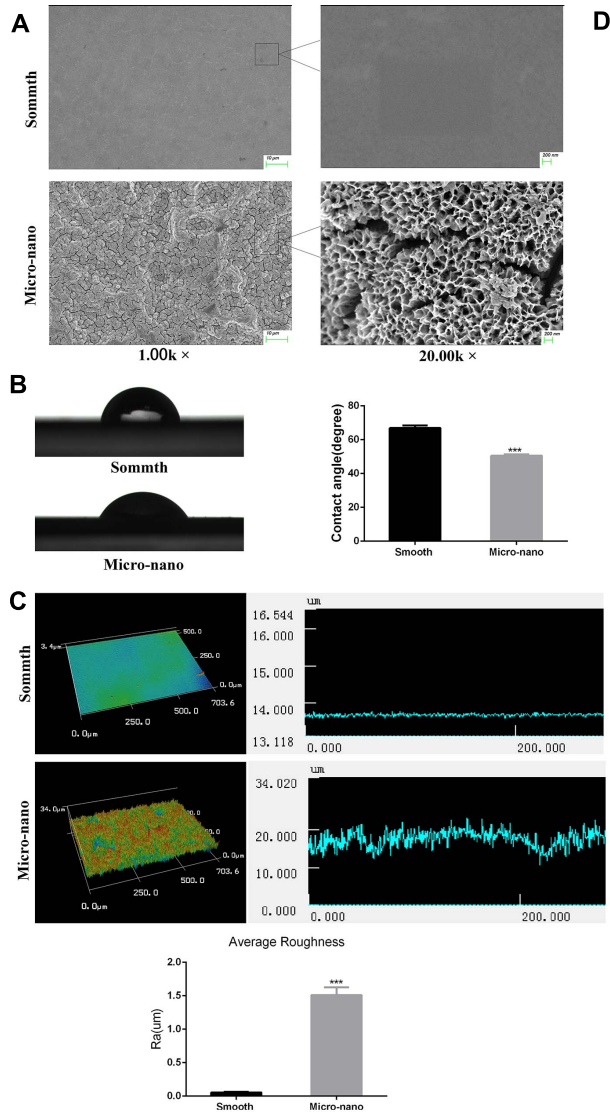

D
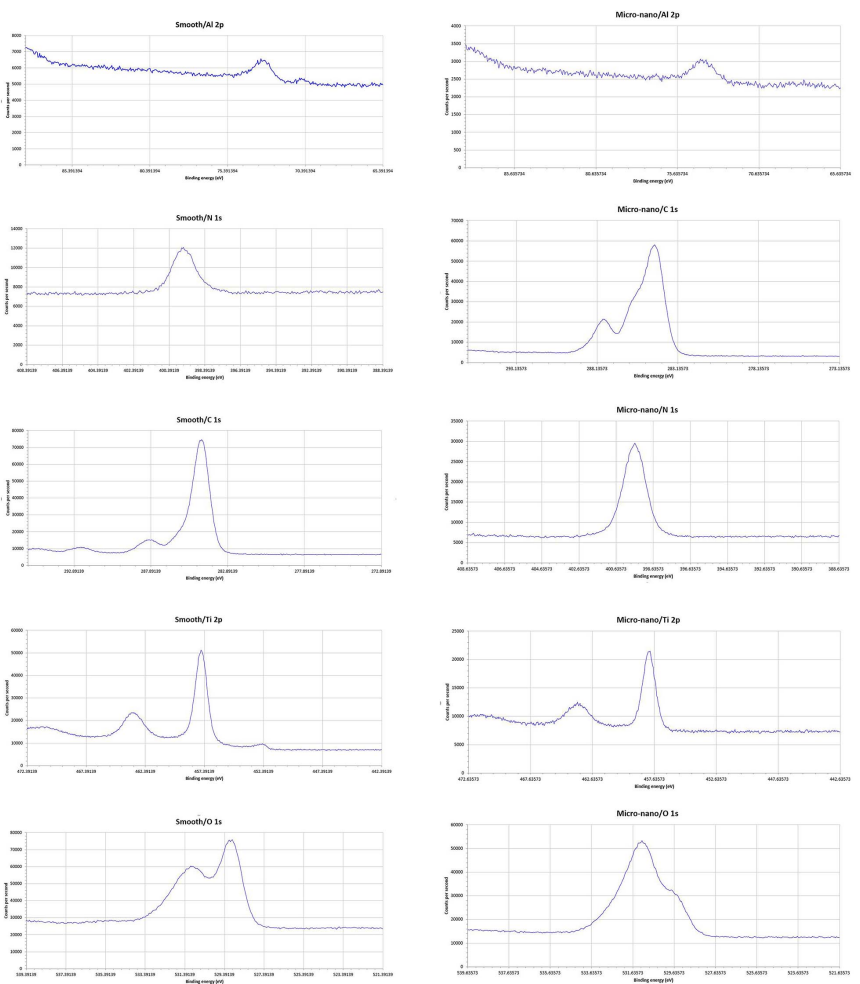

FIGURE 1 | Surface characterization of materials. (A) The surface morphology of titanium disk was observed by using a scanning electron microscope. (B) Contact angle test. (C) Three-dimensional structure diagram observation and roughness test (Yang et al., 2020). (D) XPS analysis (smooth: smooth titanium disk; micro-nano: small-scale topography titanium disk. $\left.{ }^{\star * \star} p<0.001\right)$.

database sequences (excluding miRNA), such as mRNA database, Rfam database (including rRNA, tRNA, snRNA, snoRNA, etc.) and RepBase database (repetitive sequence database), and filtered. Finally, the data obtained were valid. MicroRNA data analysis software Acgt101 Mir Program (LC Sciences, Houston, Texas, United States) was used to analyze the differentially expressed miRNAs.

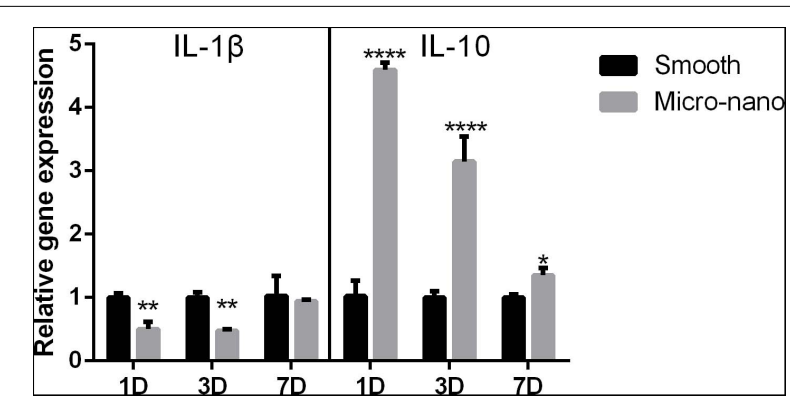

FIGURE 2 | Gene expression of RAW264.7 cells on different titanium disks. (smooth, smooth titanium disk; micro-nano, small-scale topography titanium disk. ${ }^{*} p<0.05,{ }^{\star *} p<0.01,{ }^{* \star * \star} p<0.0001$ ).

\section{Bioinformatics Analysis}

The number of genes annotated for each function or pathway corresponding to the target genes which were corresponded to the differentially expressed miRNAs was counted, and then the hypergeometric test was applied to determine the number of genes in the Gene ontology (GO) and Kyoto Encyclopedia of Genes and Genomes (KEGG) database corresponding to the target gene mRNAs which were corresponded to all the selected microRNAs. Compared all the gene in the database above (all genes with functional annotation, or all miRNA target genes with functional annotation), the significantly enriched genes or pathways were selected under the standard that $p$-value less than or equal to 0.05 . The formula for calculating the $p$-value is as

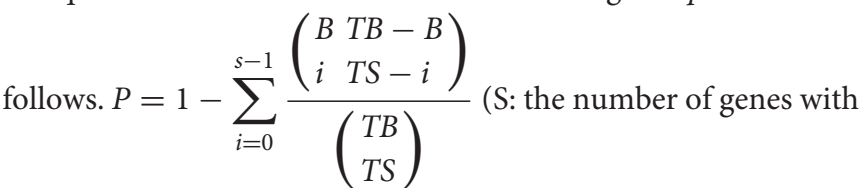
significant differences matched to a single GO/KEGG; TS: the total number of genes with significant differences; B: number of genes matched to a single GO/KEGG; TB: total number of genes.) We used the tool available on this link to draw: https: //www.omicstudio.cn/tool?order=complex. 

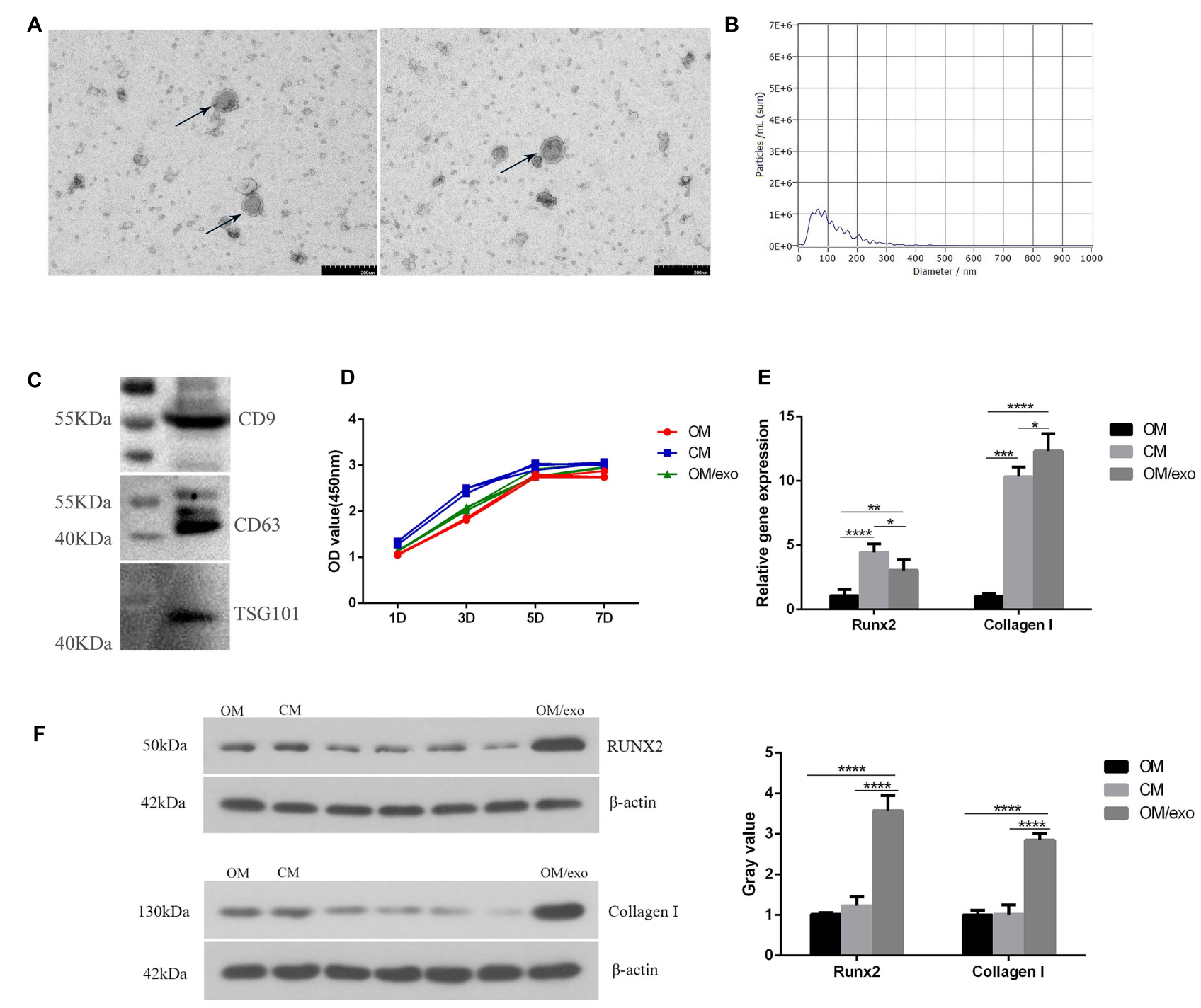

G
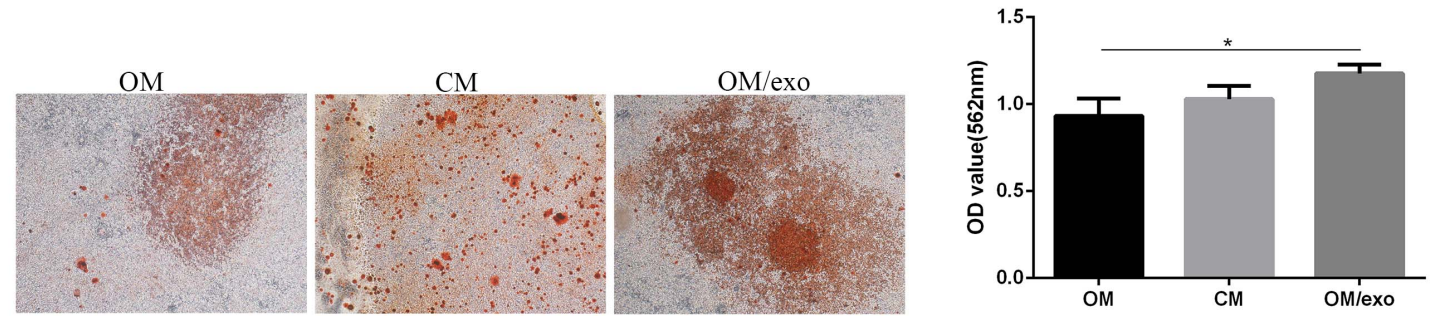

FIGURE 3 | Identification of exosomes and their effects on osteogenic differentiation of MC3T3-E1 cells. (A) TEM showed the shape of exosomes; (B) NTA showed the diameter of exosomes; (C) Western blot showed the expression of exosomes surface marker protein CD9, CD63, and TSG101; (D) CCK-8 showed cell proliferation; (E) qRT-PCR showed the expression of osteogenic related genes Runx2, Collagen I in MC3T3-E1 cells; (F) Western blot showed the expression of osteogenic related genes Runx2 and Collagen I in MC3T3-E1; (G) The mineralization of MC3T3-E1 cells was detected by alizarin red. [OM, osteogenic induction medium; CM (osteogenic induction medium + RAW264.7 cell culture medium), OM/exo: CM with RAW264.7 cell-derived exosomes ( ${ }^{*} p<0.05$; ${ }^{* *} p<0.01$; $\left.\left.{ }^{\star \star \star} p<0.001 ;{ }^{* \star \star \star} p<0.0001\right)\right]$.

\section{Statistical Analysis}

Quantitative results are expressed as mean \pm standard deviation (SD). The experiment was repeated independently at least three times. Univariate analysis of variance and multiple t-tests were used for statistical evaluation, and $p<0.05$ was set as a statistically significant threshold. 
A

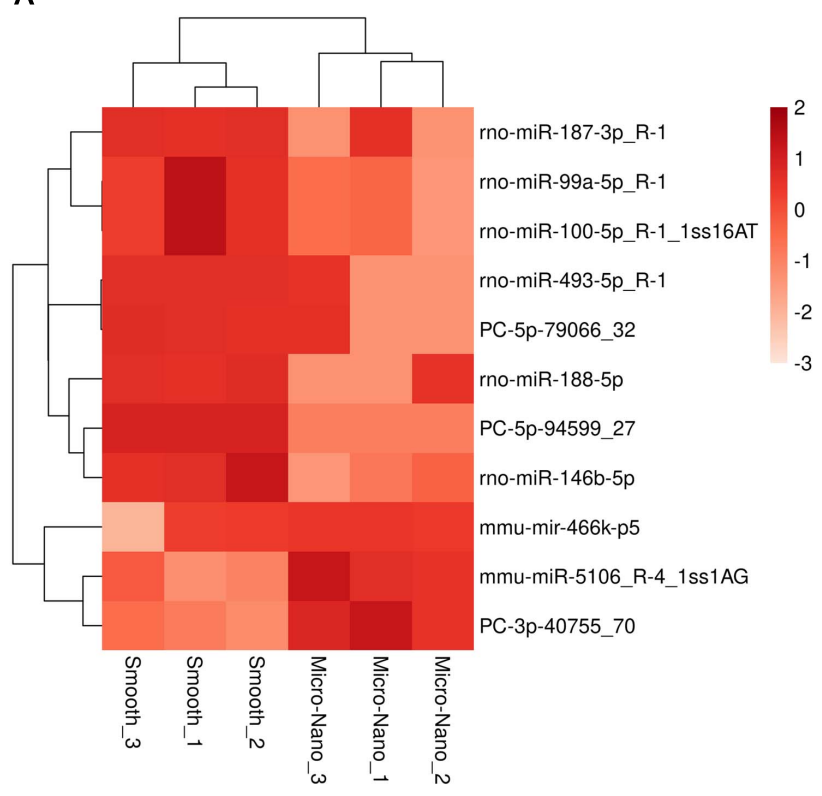

\section{B}

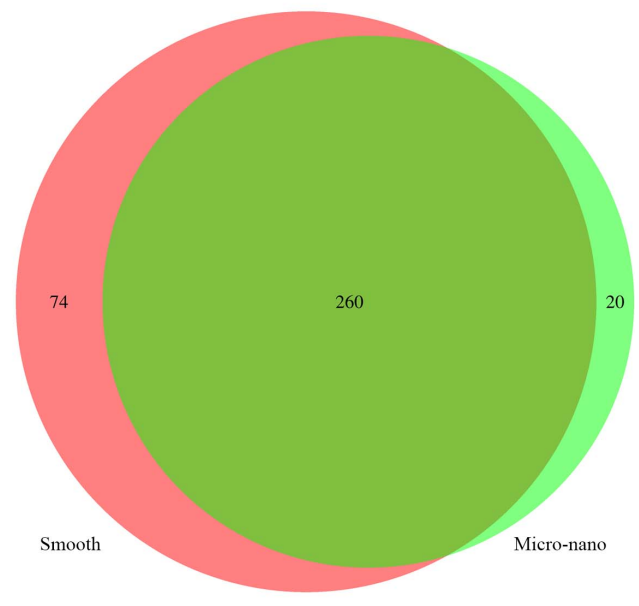

FIGURE 4 | Differentially expressed microRNAs were detected in RAW264.7 cell-derived exosomes stimulated by smooth and small-scale topography titanium disks. (A) The heat map of differentially expressed exosome miRNAs; (B) Venn map showed that there were 260 common miRNAs and 20 specific miRNAs in the differentially expressed miRNAs.

\section{RESULTS}

\section{Surface Characterization of Titanium Disks}

Figure 1A and Supplementary File 2 exhibits that the surface of smooth titanium disk is flat, without scratches; under the low power microscope, the surface of small-scale topography titanium disk is rough, no residual alumina particles are found, and a large number of pits can be seen. The pits are in the form of a multilevel continuous superposition. The secondary depression with a diameter of $2-8 \mu \mathrm{m}$ is superimposed on the surface of the first depression with a diameter of 10-50 $\mu \mathrm{m}$, and nanopores with a diameter of approximately $50-200 \mathrm{~nm}$ can be viewed under a high-power microscope.

Figure $1 \mathrm{~B}$ shows the surface droplet morphology and average surface hydrophilicity of the two types of titanium disks. Using $2 \mu \mathrm{L}$ artificial saliva as a wetting agent, the contact angle of the small-scale topography titanium disk was significantly lower than that of the smooth titanium disk $(p<0.05)$. The superficial $3 \mathrm{D}$ microstructure and roughness are shown in Figure 1C. The average surface roughness, $\mathrm{Ra}$, of the two kinds of titanium disks was further observed and compared using an optical profiler. In comparison with the smooth titanium disk, the average surface roughness of small-scale topography titanium disk increased significantly $(p<0.05$; Figure 1C, Supplementary File 3), which is consistent with the results of scanning electron microscope (Yang et al., 2020). The analysis results of the surface composition of the titanium disc are shown in the Figure 1D and Supplementary Files $4, \mathbf{5}$. All the results above proved that the titanium disk obtained by SLA technology combined with alkali heat treatment was small-scale topography, which increased the surface roughness of the titanium disk and was more conducive to osseointegration.

\section{Small-Scale Topography Titanium Disk Induces Macrophages to Polarize to Anti-inflammatory M2 Type}

After RAW264.7 cells were cultured on the surface of smooth and small-scale topography titanium disks, the expression of inflammation-related genes was detected (Figure 2). Compared to that on the surface of the smooth titanium disks, the expression level of the anti-inflammatory related gene IL-10 on the surface of small-scale topography titanium disk was increased and the expression level of the pro-inflammatory related gene IL-1 $\beta$ was decreased, with statistical significance, which was consistent with our previous research (Yang et al., 2020), and proved that smallscale topography titanium disk could stimulate the differentiation of RAW264.7 cells into M2 type to form an anti-inflammatory immune microenvironment.

\section{M2 Type RAW264.7 Cells Can Induce Osteogenic Differentiation of MC3T3-E1 Cells, and Exosomes May Play a Key Role in It}

In order to study the effect of the osteoimmune microenvironment formed by M2 type RAW264.7 cells on MC3T3-E1 cells, we co-cultured the two types of cells. The CCK-8 result showed that compared with OM, CM could promote MC3T3-E1 cells proliferation better (Figure 3D). 
A

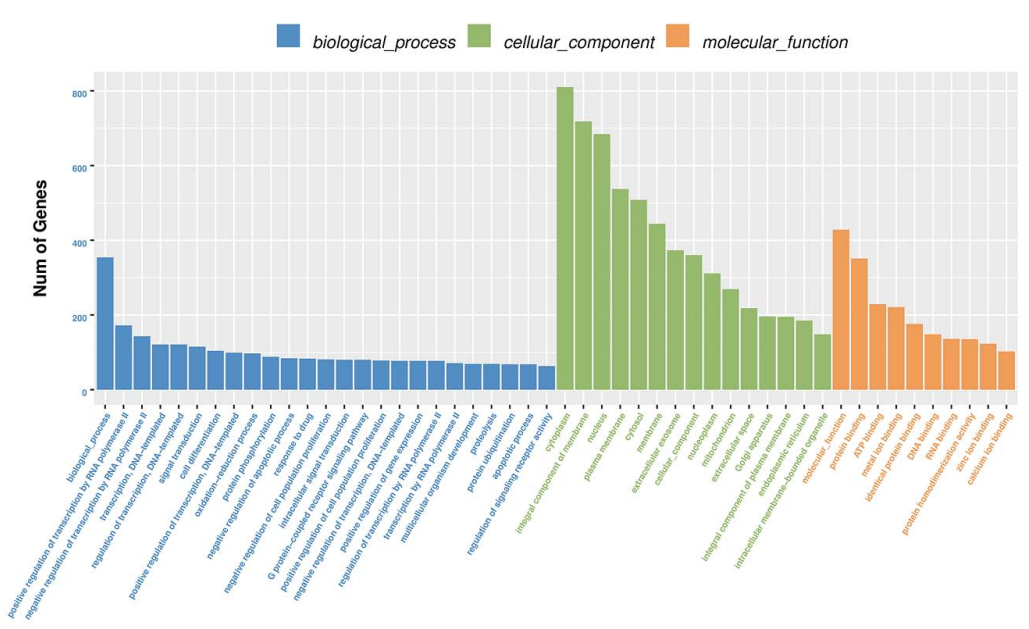

B

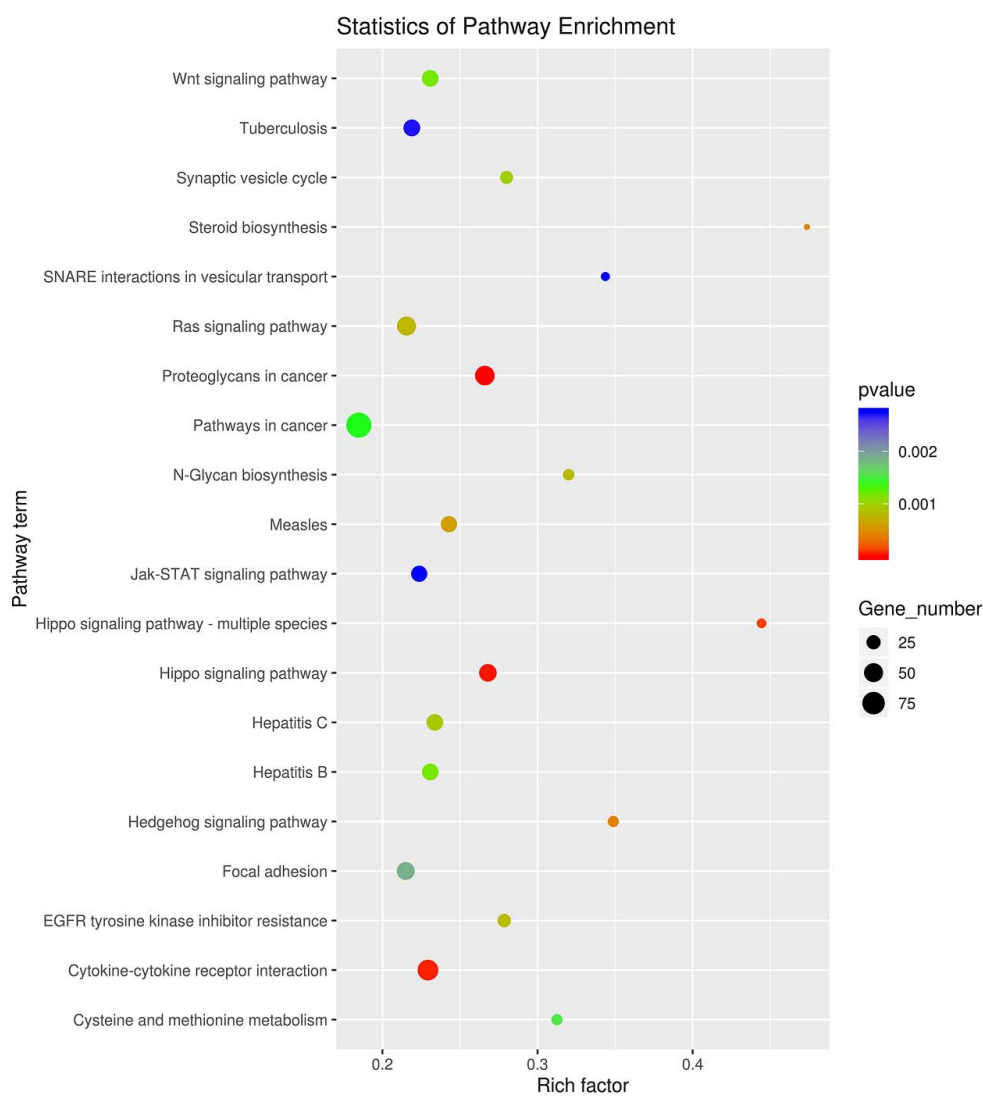

FIGURE 5 | GO analysis and Kyoto Encyclopedia of Genes and Genomes (KEGG) pathway analysis. (A) GO analysis enrichment map of biological process, cell composition and molecular function; (B) KEGG pathway analysis enrichment map (Blue means $0.002<p<0.05$; Green means $0.002<p<0.001$; Red means $p<0.001)$.

The qRT-PCR and Western blot results showed that compared with $\mathrm{OM}, \mathrm{CM}$ enhanced the expression of Collagen I and Runx2 in MC3T3-E1 cells (Figures 3E,F). AND the alizarin red staining and semi-quantitative studies showed that the mineral deposition of cells of CM was increased (Figure 3G). We speculated that the exosomes played an important role in the process that osteoimmunity promoted the osteogenic differentiation of MC3T3-E1 cells. Therefore, the exosomes derived from RAW264.7 cells stimulated by the two kinds of titanium disks were separated and studied.

First, we characterized the exosomes derived from RAW264.7 cell. Transmission electron microscopy (TEM) results showed 
that the separated vesicles had a round bilayered membrane structure (Figure 3A). NTA results showed that the peak diameter of vesicles was $63.3 \mathrm{~nm}$, accounting for $64.9 \%$ of the total area. The average diameter of vesicles was $117.1 \mathrm{~nm}$, the distribution of vesicles was in the range of 30-200 nm (Figure 3B); the expression of exosome surface proteins CD9, CD63 and TSG101 was detected by western blot (Figure 3C); the above results demonstrated that the vesicles isolated from RAW264.7 cells were exosomes.

Next, we studied the effect of the exosomes on osteogenic differentiation of MC3T3-E1 cells. The CCK-8 assay was used to study the effect of cell proliferation (Figure 3D). In comparison with the control group, exosomes promoted the proliferation of MC3T3-E1 cells, but the effect was not as pronounced as that of the CM. qRT-PCR and western blotting were used to detect the expression of osteogenesis-related marker genes and proteins (Figures 3E,F). The results showed that compared to $\mathrm{OM}$ and $\mathrm{CM}$, the expression of Collagen I and Runx2 in exosome-stimulated MC3T3-E1cells had significantly increased. Alizarin red staining and semi-quantitative studies showed that the effect of exosomes on promoting extracellular mineralization was similar to that of the MC3T3-E1 cells in CM (Figure 3G). The above studies had proved that exosomes played a key role in the process of oateoimmunity to promote osseointegration.

\section{Differentially Expressed MicroRNA Detected in RAW264.7 Cell-Derived Exosomes Stimulated by Smooth Titanium Disk and Small-Scale Topography Titanium Disk}

By RNA sequencing, we analyzed the differentially expressed microRNAs in RAW264.7 cell-derived exosomes stimulated by two kinds of titanium disks. The abscissa of the heat map represents the sample cluster, and the ordinate represents the gene cluster (Figure 4A). A total of 260 mature miRNAs and 20 specific miRNAs were expressed in RAW264.7 cell-derived exosomes (Figure 4B). Exosome microRNAs can regulate related signaling pathways by targeting downstream target genes, thereby affecting successful differentiation. According to further GO and KEGG pathway analyses, differentially expressed miRNAs are involved in most biological processes, and are mainly regulated in the Hippo signaling pathway, Wnt signaling pathway, and mTOR signaling pathway (Figures 5A,B). Table 2 summarizes and lists the 11 upregulated and downregulated miRNAs and generates the regulatory network of these 11 miRNAs (Figure 6). Some genes involved in cell proliferation and differentiation are involved, which helps to further clarify the mechanism of differential expression of microRNAs in regulating osteoimmunity and promoting osseointegration.

\section{DISCUSSION}

In this study, we used titanium disks to stimulate RAW264.7 cells to polarize into anti-inflammatory M2 phenotype, and isolated exosomes from RAW264.7 cells. We found that exosomes induced osteogenic differentiation, and mineralization of MC3T3-E1 cells. Meanwhile, we summarized the expression of microRNA of RAW264.7 cell-derived exosomes, which could provide a basis for exploring the mechanism of RAW264.7 cell-derived exosomes involved in the osteogenic differentiation of MC3T3-E1 cells.

Titanium and titanium alloys are the most widely used dental implant materials in clinics. The surface morphology of the implant has an important influence on osseointegration. A large number of studies have shown that the small-scale topography of the implant surface is more conducive to the biological behavior of osteoblasts than the simple micro-or nano- morphology (Wang et al., 2012, 2016; Ren et al., 2018). Micro-morphology can improve the mechanical properties of the implant by increasing the surface area of the implant and enhancing the mechanical chimerism between the implant and the bone cells (Dohan Ehrenfest et al., 2010). Nanomorphology can regulate the behavior of the implant by regulating the information transmission between cells (Chen et al., 2018). Compared to the traditional SLA technology, smallscale topography has a stronger ability to form hydroxyapatite in vitro, which can better promote the adhesion and extension of bone cells and promote osteogenesis cell proliferation and differentiation (Wang et al., 2012, 2016; Ren et al., 2018; Yang et al., 2020).

TABLE 2 | Differentially expressed microRNAs.

\begin{tabular}{|c|c|c|c|c|}
\hline miR name & miR seq & Log2 ratio & Up/down & $P$-value \\
\hline mmu-mir-466k-p5 & GTGTGTGTGTGTGTGTGTG & 2.02 & Up & 1.36E-02 \\
\hline PC-5p-94599_27 & GCATTGCCGGGTAGCTAA & -inf & Down & 8.77E-03 \\
\hline rno-miR-493-5p_R-1 & TTGTACATGGTAGGCTTCAT & -2.72 & Down & 1.65E-02 \\
\hline rno-miR-146b-5p & TGAGAACTGAATTCCATAGGCTGT & -1.31 & Down & 2.14E-02 \\
\hline rno-miR-100-5p_R-1_1ss16AT & AACCCGTAGATCCGATCTTGT & -0.27 & Down & 3.27E-02 \\
\hline PC-5p-79066_32 & GAGGAGTACTAGTCGGCA & -2.35 & Down & 3.61E-02 \\
\hline rno-miR-188-5p & САTCCCTTGCATGGTGGAGGG & -2.72 & Down & 4.16E-02 \\
\hline rno-miR-187-3p_R-1 & TCGTGTCTTGTGTTGCAGCCG & -2.24 & Down & 4.79E-02 \\
\hline
\end{tabular}




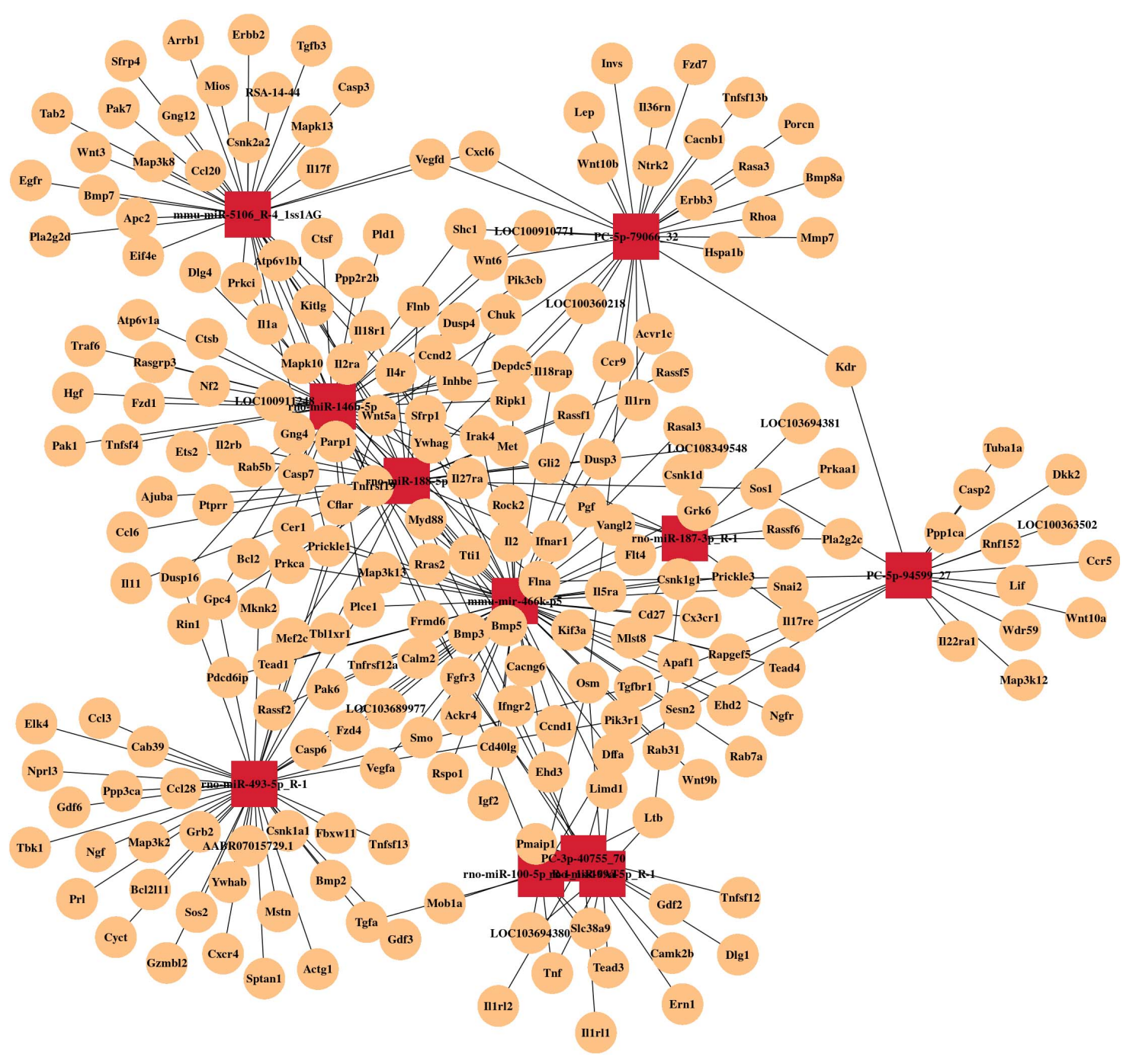

FIGURE 6 | MicroRNA mRNA network. The relationship between differentially expressed miRNAs and their target genes.

During osseointegration, there is a close relationship between the immune system and skeletal system. The immune system plays a key role in tissue repair and regeneration (Forbes and Rosenthal, 2014); macrophages and myeloid heterogeneous immune cells play an important role in the process of osseointegration. They can polarize to the pro-inflammatory M1 phenotype or anti-inflammatory M2 phenotype according to different stimuli (Gordon and Taylor, 2005; Gordon and Martinez, 2010; Michalski and McCauley, 2017). Many studies have shown that immune cells can be affected by various factors of the implant, including surface morphology (Fink et al., 2008; Spiller et al., 2009). In our previous study, we found that the small-scale topography titanium disk can stimulate RAW264.7 cells to polarize to the M2 phenotype, secrete IL-10 and VEGF, regulate the immune environment, and promote the osteogenic differentiation of MC3T3-E1 cells (Yang et al., 2020).

Exosome biogenesis is a dynamic but highly ordered process involving two invasions of the plasma membrane and the formation of intraluminal vesicles (ILVs) and intracellular multivesicular bodies (MVBs) (Li et al., 2019; Kalluri and LeBleu, 2020). Then, MVBs fuse with lysosomes to degrade, or with the plasma membrane to release ILV into the extracellular environment and become exosomes (van Niel et al., 2018; Mathieu et al., 2019), which then actively participate in the functional changes of many cells. The nature and content of exosomes are cell type-specific (Kalra et al., 2016), which are usually influenced by the physiological or pathological state of donor cells, the stimulation, and the molecular mechanism of biogenesis (Minciacchi et al., 2015). Studies have shown that many cell types and molecular mechanisms contribute to the coupling between bone resorption and bone formation (Sims and Martin, 2014). Exosomes from mononuclear phagocytes are most likely to play a role in maintaining bone homeostasis (Silva et al., 2017). Belonging to Runx family, expressed in osteoblasts, Runx2 is responsible for activating osteoblast differentiation marker genes, and plays a key role in the process of osteogenic 
differentiation (Vimalraj et al., 2015). Ekström et al. (2013) showed that LPS-stimulated monocytes can communicate with mesenchymal stem cells through exosomes, which can increase the expression of Runx 2 and BMP-2 in mesenchymal stem cells. This is considered to be an intercellular signal transduction mode from the stages of injury and inflammation till bone regeneration. Qin et al. (2016) confirmed that the secretion of human bone marrow mesenchymal stem cells can effectively promote the proliferation and differentiation of rat bone marrow mesenchymal stem cells. Qi et al. (2016) combined human induced pluripotent stem cell-derived exosomes with $\beta$-tricalcium phosphate $(\beta$-TCP) scaffolds to repair rat skull defects. It was found that the repair effect of the exosome composite scaffolds was significantly better than that of the $\beta$-TCP scaffolds alone, and exosomes could promote the osteogenic differentiation of bone marrow mesenchymal stem cells by activating the PI3K/Akt signaling pathway. In this study, exosomes derived from M2 RAW264.7 cells induced upregulation of Runx2 and Collagen I expression in MC3T3E1 cells, and the effect was significantly better than that in the macrophage co-culture group, indicating that exosomes play an important role in the process of osteoimmunity-promoting osseointegration. At the same time, we found that three miRNAs were upregulated and eight miRNAs were downregulated in M2 RAW264.7 cell-derived exosomes, and the corresponding target genes involved in the regulation of multiple signaling pathways, such as mTOR, AMPK and Wnt signaling pathways, which play an important role in the process of osseointegration. After RNA sequencing of exosomes derived from hMSCs induced by osteogenesis, Zhai et al. (2020) found that exosomes induce osteogenic differentiation through microRNA, among which the miRNAs HAS-Mir-146a-5p, HAS-Mir-503-5p, HAS-Mir483-3P, and HAS-MIR) that contribute to bone formation. The upregulation of HAS-Mir-32-5p, HAS-Mir-133a-3P, and HAS-Mir-204-5p activated the PI3K/Akt and MAPK signaling pathways related to osteogenesis.

\section{CONCLUSION}

In conclusion, we successfully isolated exosomes from RAW264.7 cells, which were induced to polarize to the M2 phenotype by preparing small-scale topography titanium disks. After co-culture with MC3T3-E1 cells, we found that exosomes significantly promoted the osteogenic differentiation and mineralization of MC3T3-E1 cells. Through RNA sequencing and gene analysis, we found differentially expressed microRNAs

\section{REFERENCES}

Chen, S., Guo, Y., Liu, R., Wu, S., Fang, J., Huang, B., et al. (2018). Tuning surface properties of bone biomaterials to manipulate osteoblastic cell adhesion and the signaling pathways for the enhancement of early osseointegration. Colloids Surf B Biointerfaces 164, 58-69. doi: 10.1016/j.colsurfb.2018.01.022

Dar, H. Y., Azam, Z., Anupam, R., Mondal, R. K., and Srivastava, R. K. (2018), Osteoimmunology: the nexus between bone and immune system. Front. Biosci. 23:464-492. doi: $10.2741 / 4600$ that targeted the signal pathways that may be related, such as mTOR, AMPK, Wnt, etc., and thus provide a reference for the mechanism of osteoimmunue regulation of implant osseointegration. The deficiency of this study is that the selection of RAW264.7 cells and MC3T3-E1 cells has certain limitations, and the related research is still insufficient. In the next research, we will focus on the exosome microRNA and its downstream key factors, and further study the molecular mechanism of osteoimmune effect on small-scale topography implant osseointegration through exosomes.

\section{DATA AVAILABILITY STATEMENT}

The datasets presented in this study can be found in online repositories. The names of the repository/repositories and accession number(s) can be found below: GSE175428, https:// www.ncbi.nlm.nih.gov/geo/query/acc.cgi?acc=GSE175428.

\section{AUTHOR CONTRIBUTIONS}

HS, PY, and TZ conceived the project and designed experiments. TZ, MJ, and XY performed the experiments. TZ and MJ analyzed the data. TZ wrote the manuscript. All authors commented on the manuscript.

\section{FUNDING}

This work was funded and supported by the Key Research and Development Program of Shandong Province (2019GSF107001), Jinan Clinical Medical Science and Technology Innovation Plan (202019167), and Laboratory Construction and Management Research Project of Shandong University (sy20212401).

\section{ACKNOWLEDGMENTS}

Thanks to the School of Mechanical Engineering, Shandong University for providing technical support for this research.

\section{SUPPLEMENTARY MATERIAL}

The Supplementary Material for this article can be found online at: https://www.frontiersin.org/articles/10.3389/fbioe. 2021.682384/full\#supplementary-material

Dohan Ehrenfest, D. M., Coelho, P. G., Kang, B. S., Sul, Y. T., and Albrektsson, T. (2010). Classification of osseointegrated implant surfaces: materials, chemistry and topography. Trends Biotechnol. 28, 198-206.

Ekström, K., Omar, O., Granéli, C., Wang, X., Vazirisani, F., and Thomsen, P. (2013). Monocyte exosomes stimulate the osteogenic gene expression of mesenchymal stem cells. PloS One 8:e75227. doi: 10.1371/journal.pone.0075227

Fink, J., Fuhrmann, R., Scharnweber, T., and Franke, R. P. (2008). Stimulation of monocytes and macrophages: possible influence of surface roughness. Clin. Hemorheol. Microcirc. 39, 205-212. 
Forbes, S. J., and Rosenthal, N. (2014). Preparing the ground for tissue regeneration: from mechanism to therapy. Nat. Med. 20, 857-869. doi: 10.1038/ nm.3653

Gordon, S., and Martinez, F. O. (2010). Alternative activation of macrophages: mechanism and functions. Immunity 32, 593-604. doi: 10.1016/j.immuni.2010. 05.007

Gordon, S., and Taylor, P. R. (2005). Monocyte and macrophage heterogeneity. Nat. Rev. Immunol. 5, 953-964. doi: 10.1038/nri1733

Kalluri, R., and LeBleu, V. S. (2020). The biology, function, and biomedical applications of exosomes. Science 367:eaau6977. doi: 10.1126/science.aau6977

Kalra, H., Drummen, G. P., and Mathivanan, S. (2016). Focus on extracellular vesicles: introducing the next small big thing. Int. J. Mol. Sci. 17:170. doi: 10.3390/ijms 17020170

Li, Q., Wang, H., Peng, H., Huyan, T., and Cacalano, N. A. (2019). Exosomes: versatile nano mediators of immune regulation. Cancers 11:1557. doi: 10.3390/ cancers 11101557

Loi, F., Córdova, L. A., Pajarinen, J., Lin, T. H., Yao, Z., and Goodman, S. B. (2016). Inflammation, fracture and bone repair. Bone 86, 119-130. doi: 10.1016/j.bone. 2016.02.020

Luu, T. U., Gott, S. C., Woo, B. W., Rao, M. P., and Liu, W. F. (2015). Micro- and nanopatterned topographical cues for regulating macrophage cell shape and phenotype. ACS Appl. Mater. Interfaces 7, 28665-28672. doi: 10.1021/acsami. 5 b10589

Mathieu, M., Martin-Jaular, L., Lavieu, G., and Théry, C. (2019). Specificities of secretion and uptake of exosomes and other extracellular vesicles for cell-to-cell communication. Nat. Cell Biol. 21, 9-17. doi: 10.1038/s41556-018-0250-9

Michalski, M. N., and McCauley, L. K. (2017). Macrophages and skeletal health. Pharmacol. Ther. 174, 43-54. doi: 10.1016/j.pharmthera.2017.02.017

Minciacchi, V. R., Freeman, M. R., and Di Vizio, D. (2015). Extracellular vesicles in cancer: exosomes, microvesicles and the emerging role of large oncosomes. Semin. Cell Dev. Biol. 40, 41-51. doi: 10.1016/j.semcdb.2015.02.010

Miron, R. J., and Bosshardt, D. D. (2016). OsteoMacs: key players around bone biomaterials. Biomaterials 82, 1-19. doi: 10.1016/j.biomaterials.2015.12.017

Pan, B. T., and Johnstone, R. M. (1983). Fate of the transferrin receptor during maturation of sheep reticulocytes in vitro: selective externalization of the receptor. Cell 33, 967-978. doi: 10.1016/0092-8674(83)90040-5

Pieters, B., Cappariello, A., van den Bosch, M., van Lent, P., Teti, A., and van de Loo, F. (2019). Macrophage-derived extracellular vesicles as carriers of alarmins and their potential involvement in bone homeostasis. Front. Immunol. 10:1901. doi: 10.3389/fimmu.2019.01901

Qi, X., Zhang, J., Yuan, H., Xu, Z., Li, Q., Niu, X., et al. (2016). Exosomes secreted by human-induced pluripotent stem cell-derived mesenchymal stem cells repair critical-sized bone defects through enhanced angiogenesis and osteogenesis in osteoporotic rats. Int. J. Biol. Sci. 12, 836-849. doi: 10.7150/ijbs.14809

Qin, Y., Wang, L., Gao, Z., Chen, G., and Zhang, C. (2016). Bone marrow stromal/stem cell-derived extracellular vesicles regulate osteoblast activity and differentiation in vitro and promote bone regeneration in vivo. Sci. Rep. 6:21961. doi: 10.1038/srep21961

Quan, H., Kim, Y., Wu, L., Park, H. C., and Yang, H. C. (2020). Modulation of macrophage polarization by phospholipids on the surface of titanium. Molecules 25:2700. doi: 10.3390/molecules25112700

Rani, S., Ryan, A. E., Griffin, M. D., and Ritter, T. (2015). Mesenchymal stem cellderived extracellular vesicles: toward cell-free therapeutic applications. Mol. Ther. 23, 812-823. doi: 10.1038/mt.2015.44

Ren, B., Wan, Y., Wang, G., Liu, Z., Huang, Y., and Wang, H. (2018). Morphologically modified surface with hierarchical micro-/nano-structures for enhanced bioactivity of titanium implants. J. Mater. Sci. 53, 12679-12691. doi: 10.1007/s10853-018-2554-3
Silva, A. M., Teixeira, J. H., Almeida, M. I., Gonçalves, R. M., Barbosa, M. A., and Santos, S. G. (2017). Extracellular vesicles: immunomodulatory messengers in the context of tissue repair/regeneration. Eur. J. Pharm. Sci. 98, 86-95. doi: 10.1016/j.ejps.2016.09.017

Sims, N. A., and Martin, T. J. (2014). Coupling the activities of bone formation and resorption: a multitude of signals within the basic multicellular unit. Bonekey Rep. 3:481. doi: 10.1038/bonekey.2013.215

Spiller, D., Mirtelli, C., Losi, P., Briganti, E., Sbrana, S., Counoupas, S., et al. (2009). In vitro evaluation of the PEtU-PDMS material immunocompatibility: the influence of surface topography and PDMS content. J. Mater. Sci. Mater. Med. 20, 2511-2520. doi: 10.1007/s10856-009-3823-0

van Niel, G., D'Angelo, G., and Raposo, G. (2018). Shedding light on the cell biology of extracellular vesicles. Nat. Rev. Mol. Cell Biol. 19, 213-228. doi: $10.1038 / \mathrm{nrm} .2017 .125$

Vimalraj, S., Arumugam, B., Miranda, P. J., and Selvamurugan, N. (2015). Runx2: structure, function, and phosphorylation in osteoblast differentiation. Int. J. Biol. Macromol. 78, 202-208. doi: 10.1016/j.ijbiomac.2015.04.008

Wang, T., Wan, Y., and Liu, Z. (2016). Fabrication of hierarchical micro/nanotopography on bio-titanium alloy surface for cytocompatibility improvement. J. Mater. Sci. 51, 9551-9561. doi: 10.1007/s10853-016-0 219-7

Wang, W., Zhao, L., Ma, Q., Wang, Q., Chu, P. K., and Zhang, Y. (2012). The role of the Wnt/ $\beta$-catenin pathway in the effect of implant topography on MG63 differentiation. Biomaterials 33, 7993-8002. doi: 10.1016/j.biomaterials.2012. 07.064

Wei, F., Li, M., Crawford, R., Zhou, Y., and Xiao, Y. (2019). Exosome-integrated titanium oxide nanotubes for targeted bone regeneration. Acta Biomater. 86, 480-492. doi: 10.1016/j.actbio.2019.01.006

Xiong, Y., Chen, L., Yan, C., Zhou, W., Yu, T., Sun, Y., et al. (2020). M2 macrophagy-derived exosomal miRNA-5106 induces bone mesenchymal stem cells towards osteoblastic fate by targeting salt-inducible kinase 2 and 3 . J. Nanobiotechnol. 18:66. doi: 10.1186/s12951-020-00622-5

Xue, T., Attarilar, S., Liu, S., Liu, J., Song, X., Li, L., et al. (2020). Surface modification techniques of titanium and its alloys to functionally optimize their biomedical properties: thematic review. Front. Bioeng. Biotechnol. 8:603072. doi: $10.3389 /$ fbioe. 2020.603072

Yang, F., Liao, X., Tian, Y., and Li, G. (2017). Exosome separation using microfluidic systems: size-based, immunoaffinity-based and dynamic methodologies. Biotechnol. J. 12:1600699. doi: 10.1002/biot.2016 00699

Yang, Y., Zhang, T., Jiang, M., Yin, X., Luo, X., and Sun, H. (2020). Effect of the immune responses induced by implants in a integrated three-dimensional micro-nano topography on osseointegration. J. Biomed. Mater. Res. A 109, 1429-1440. doi: 10.1002/jbm.a.37134

Zhai, M., Zhu, Y., Yang, M., and Mao, C. (2020). Human mesenchymal stem cell derived exosomes enhance cell-free bone regeneration by altering their miRNAs profiles. Adv. Sci. 7:2001334. doi: 10.1002/advs.202001334

Conflict of Interest: The authors declare that the research was conducted in the absence of any commercial or financial relationships that could be construed as a potential conflict of interest.

Copyright (c) 2021 Zhang, Jiang, Yin, Yao and Sun. This is an open-access article distributed under the terms of the Creative Commons Attribution License (CC BY). The use, distribution or reproduction in other forums is permitted, provided the original author(s) and the copyright owner(s) are credited and that the original publication in this journal is cited, in accordance with accepted academic practice. No use, distribution or reproduction is permitted which does not comply with these terms. 